Notre Dame Journal of Formal Logic

Volume 37, Number 4, Fall 1996

\title{
A Basic System of Congruential-to-Monotone Bimodal Logic and Two of its Extensions
}

\author{
I. L. HUMBERSTONE
}

\begin{abstract}
If what is known need not be closed under logical consequence, then a distinction arises between something's being known to be the case (by a specific agent) and its following from something known (to that subject). When each of these notions is represented by a sentence operator, we get a bimodal logic in which to explore the relations between the two notions.
\end{abstract}

1 Introduction Let us begin with the threefold distinction among what is known, what is epistemically necessary (= follows from what is known), and what is epistemically possible (= is compatible with what is known). Representing these as singulary operators $K, \square_{K}$, and $\diamond_{K}$ respectively, one way for the first two to be distinct is to take $\mathrm{K}$ as monotone but not regular, in the sense of Chellas's extension ([1], [2]) of Segerberg's classification [14], with roughly and informally speaking the following interpretation: $\square_{\mathrm{K}} \alpha$ is true when $\alpha$ follows from some $\beta_{1}, \ldots, \beta_{n}$ taken together, where each $\mathrm{K} \beta_{i}$ is true. (We use 'monotone' rather than 'monotonic' to avoid confusions with monotonic vs. non-monotonic logic; and below we follow Segerberg 15 and say 'congruential' where the references cited above say 'classical'.) A second is that we assume only that $\mathrm{K}$ is congruential, and again understand $\square_{\mathrm{K}}$ in accordance with the preceding explanation. On both these options, $\square_{\mathrm{K}}$ is regular (indeed normal, if we allow $n=0) .{ }^{1}$ Thirdly, we could take $\mathrm{K}$ as merely congruential again, but count $\square_{\mathrm{K}} \alpha$ as true if $\alpha$ follows from some (one) $\beta$ for which $\mathrm{K} \beta$ is true, making $\square_{\mathrm{K}}$ monotone-but not guaranteeing regularity. We shall explore this third option here. (Actually, we consider a "propositional" rather than a "sentential" version of the possibility. This distinction is explained in the following section.)

After the present section, there will be no further need to pay attention separately to $\diamond_{\mathrm{K}}$, since we may take $\diamond_{\mathrm{K}} \alpha$ as abbreviating $\neg \square_{\mathrm{K}} \neg \alpha$. But we remark here that corresponding to the distinction between what is known and what is epistemically necessary is the dual distinction between $\diamond_{\mathrm{K}} \alpha$ and $\neg \mathrm{K} \neg \alpha$ (or $P \alpha$, in the notation of Hintikka [6]), each of which has some claim to be regarded as embodying a notion of

Received November 8, 1995; revised September 6, 1996 
epistemic possibility, the opening sentence above notwithstanding. We may illustrate the distinction between them as it arises on what we have called the third option, by considering the treatment of an example (originally due to E. Wolgast) presented by the non-monotone epistemologist Oswald Hanfling (44], pp. 442-4). You remember where you parked your car this morning and on that basis claim to know that your car is in the car park; the latter proposition entails that the car has not been removed from the car park by car thieves since you left it there this morning-for brevity, that it has not been stolen-yet you do not know, illustrating the failure of the monotony condition, that the car has not been stolen (having done nothing expressly to rule out this possibility). Hanfling holds that although you don't know that the car has not been stolen, you may legitimately take this for granted in claiming to know that your car is in the car park. Controversial though it may be, suppose we accept the above commentary on the example. ${ }^{2}$ Is it or is it not epistemically possible (for you) that your car has been stolen? On the one hand, you know something, namely, that your car is in the car park, from which it follows that the car has not been stolen. On the other hand, ex hypothesi, you do not know that it hasn't been stolen. Whatever answer is given to the question about how to use the label 'epistemically possible' (or for that matter, about how to answer the question of whether for all you know the car hasn't been stolen), the need to distinguish between $\diamond_{\mathrm{K}} \alpha$ and $\neg \mathrm{K} \neg \alpha$ on the third (or indeed the first or second) option is clear enough. ${ }^{3}$

A fourth option-and there are obviously further variations on this theme possible - is to make no assumptions about $\mathrm{K}$, and take $\square_{\mathrm{K}} \alpha$ to be true if $\alpha$ is logically equivalent to some $\beta$ for which $\mathrm{K} \beta$ is true. We will write ' $\mathrm{O}$ ' rather than ' $\mathrm{K}$ ' from now on, since our starting point may as well be an arbitrary congruential operator, and we do not wish to invite distraction from specifically epistemic concerns. ${ }^{4}$

The way we pass from $\mathrm{O}$ to $\square_{\mathrm{O}}$ on the first option above may be called 'monotone-to-regular'; on the second option, 'congruential-to-regular'; on the third and fourth, respectively, 'congruential-to-monotone' and 'unrestricted-to-congruential'. Our interest here, then, is in a bimodal congruential-to-monotone logic, and in particular, in the basic such logic given by the semantics introduced in the following section to make precise the above ideas.

2 Semantics and axioms Since we assume only that $\mathrm{O}$ is congruential, it may appropriately be given a semantic treatment in the Scott-Montague style, using models $\langle W, N, V\rangle$ on neighborhood frames $\langle W, N\rangle$; here $W$ is a nonempty set and $N$ assigns to each $x \in W$ a collection, denoted $N_{x}$, of subsets of $W ; V$ assigns subsets of $W$ to the propositional variables (sentence letters), of which we shall assume there are countably many $\left(p_{1}, \ldots, p_{n}, \ldots\right)$, and we put

$$
\langle W, N, V\rangle \models_{x} p_{i} \text { if and only if } x \in V\left(p_{i}\right)
$$

and

$$
\langle W, N, V\rangle \models_{x} \alpha \wedge \beta \text { if and only if }\langle W, N, V\rangle \models_{x} \alpha \text { and }\langle W, N, V\rangle \models_{x} \beta
$$

(and similary for other Boolean connectives, of which we assume we have a functionally complete set, also including at least $\neg$ and $\perp$ ). For $\mathrm{O}$ we say

$$
\langle W, N, V\rangle \models_{x} \mathrm{O} \alpha \text { if and only if }\|\alpha\| \in N_{x},
$$


where

$$
\|\alpha\|=\left\{y \in W:\langle W, N, V\rangle \models_{y} \alpha\right\} .
$$

In the case in which $\mathrm{O}$ is thought of as representing a propositional attitude, the set $N_{x}$ accordingly represents the set of propositions (= collections of worlds) to which some fixed individual has the attitude in question. Accordingly, we want $\square_{\mathrm{O}} \alpha$ to mean that $\alpha$ follows from some such proposition, and lay down the clause

$$
\langle W, N, V\rangle \models_{x} \square_{\mathrm{O}} \alpha \text { if and only if for some } Y \in N_{x}, Y \subseteq\|\alpha\| .
$$

Here is where the parenthetical remark from Section 1 comes in, about opting for a propositional rather than a sentential interpretation of the idea that $\square_{\mathrm{O}} \alpha$ means that $\alpha$ follows from something to which the agent has the propositional attitude registered by $O$. In the case discussed in the introduction, we want $\square_{\mathrm{K}} \alpha$ to mean that the truth of $\alpha$ follows something our agent knows. There, for expository convenience, we glossed this as meaning that for some sentence (or formula) $\beta$ for which $\mathrm{K} \beta$ is true, $\alpha$ follows from $\beta$ : a specifically sentential construal. Here, in accordance with the remarks preceding the above clause for $\square_{\mathrm{O}}$, we are adopting a propositional construal in not insisting that the proposition schematically indicated by the ' $Y$ ' in that clause should be linguistically expressible. (The sentential version of that clause would have on its right-hand side: for some $\beta$ such that $\|\beta\| \in N_{x},\|\beta\| \subseteq\|\alpha\|$.)

As usual, a formula is valid on a frame if it is true at every point in every model on that frame (with the above clauses constituting the inductive definition of truth at a point in a model). We call a schema valid on $\langle W, N\rangle$ when every instance of the schema is valid on $\langle W, N\rangle$. The problem we consider is: How do we axiomatize the class of formulas valid on every frame? Since this class includes all the truthfunctional tautologies and their substitution instances in the present language, and is closed under tautological consequence, we list explicitly only those modal principles required in addition to any complete truth-functional basis. Two such principles were already covered in our informal introduction-congruentiality for $\mathrm{O}$ and monoton(icit)y for $\square_{\mathrm{O}}$, namely, the following rules.

$$
\begin{array}{cl}
\text { (Cong. O) } & \text { From } \vdash \alpha \longleftrightarrow \beta \text { to } \vdash \mathrm{O} \alpha \longleftrightarrow \mathrm{O} \beta . \\
\text { (Mon. } \left.\square_{\mathrm{O}}\right) & \text { From } \vdash \alpha \rightarrow \beta \text { to } \vdash \square_{\mathrm{O}} \alpha \rightarrow \square_{\mathrm{O}} \beta .
\end{array}
$$

These rules preserve truth in every model, and therefore preserve the property of being valid on every frame, which property is only slightly less obviously possessed by all instances of the following schemata.

$$
\begin{array}{ll}
\text { (A1) } & \mathrm{O} \alpha \rightarrow \square_{\mathrm{O}} \alpha . \\
\text { (A2) } & \square_{\mathrm{O} \perp \rightarrow \mathrm{O} \perp .}
\end{array}
$$

We make two observations concerning this axiom system. First, although we have not used Uniform Substitution as a rule of proof, the role played by schematic letters shows that the set of provable formulas is closed under this rule (a fact to be exploited below). Secondly, we should notice that because of (A2) it would be inaccurate to say that what we have here is the smallest bimodal supersystem of a congruential monomodal system in which the new operator ' $\square_{\mathrm{O}}$ ' is a monotone extension-using 'extension' in the sense of Note 4 - of the given operator ' $O$ '. 
3 Completeness of the axiomatization The axiomatization given for the logic of $\mathrm{O}$ and $\square_{\mathrm{O}}$ in the preceding section is clearly sound (with respect to the class of all frames): the provable formulas are all valid on every frame, and we address ourselves to the question of its completeness. We wish (as usual) to find an invalidating frame for every nontheorem, and do so by constructing a canonical model, $\langle W, N, V\rangle$, the frame of which behaves as desired. As in $14 \mathrm{~W}$ is to be the set of all sets of formulas which are maximally consistent (with respect to the logic axiomatized in the preceding section), and $V\left(p_{i}\right)=\left|p_{i}\right|$, where we have used (as in [1], [14]) the abbreviative notation $|\alpha|$ for $\{x \in W: \alpha \in x\}$. We define $N$ using a selection function $f$, with $f(Y) \in Y$ for all nonempty subsets $Y$ of $W(f$ is a total function, and so is defined also for $\varnothing$ as argument, but no constraint is imposed on its value for this case). Then we stipulate that for $x \in W, Y \subseteq W$, we have $Y \in N_{x}$ if and only if either

(i) for some $\alpha$ such that $\mathrm{O} \alpha \in x, Y=|\alpha|$, or

(ii) for some $\alpha$ such that $\square_{\mathrm{O}} \alpha \in x$ and $|\alpha| \neq \varnothing, Y=|\alpha| \backslash\{f(|\alpha|)\}$.

We call the sets $Y$ which satisfy the condition in (i) 'type (i) neighborhoods' of $x$, and those satisfying that in (ii), 'type (ii) neighborhoods' of $x$. (It will turn out $-\mathrm{cf}$. the remark following Lemma 3.2 below-that no $Y \subseteq W$ is of both types.) Type (i) neighborhoods are formula-definable subsets of $W$ in the sense of being sets of the form $|\alpha|$ for some $\alpha .{ }^{5}$ (Formula-definable sets are called proof sets in [1].) In the proofs below, $\vdash$ indicates provability in whatever logic is under discussion-here, the basic system axiomatized in Section 2-though Lemma 3.1 and Lemma 3.2 do not depend on the specifically modal aspects of that system.

Lemma 3.1 If $w \in W$ then $\{w\}$ is not formula-definable.

Proof: Suppose $\{w\}=|\alpha|$. Let $p_{i}$ be a propositional variable not occurring in $\alpha$. Since $\vdash \alpha \longleftrightarrow\left(\left(\alpha \wedge p_{i}\right) \vee\left(\alpha \wedge \neg p_{i}\right)\right),|\alpha|=\left|\alpha \wedge p_{i}\right| \cup\left|\alpha \wedge \neg p_{i}\right|$, so since the two terms of this union are disjoint, the only way for $|\alpha|$ to be a unit set is for one or the other of them to be empty. Suppose it is $\left|\alpha \wedge \neg p_{i}\right|$ that is empty. Then $\vdash \alpha \rightarrow p_{i}$, and so, since our logic is closed under Uniform Substitution and $p_{i}$ does not occur in $\alpha, \vdash \alpha \longrightarrow \perp$, and therefore $\vdash \alpha \longleftrightarrow \perp$, so $|\alpha|=|\perp|=\varnothing \neq\{w\}$ after all. We get a similar contradiction from supposing $\left|\alpha \wedge p_{i}\right|=\varnothing$.

In fact, by a slight variant on the above argument, one can show that no nonempty finite subset of $W$ is formula-definable. This is not something we need for the following developments, however.

Lemma 3.2 If $w \in|\alpha|$, then $|\alpha| \backslash\{w\}$ is not formula-definable.

Proof: Suppose $w \in|\alpha|$ and $|\beta|=|\alpha| \backslash\{w\}$. Then $\{w\}=|\alpha \wedge \neg \beta|$, contradicting Lemma3.1.

The point of Lemma 3.2 will emerge in the proof of our main result (Theorem 3.4, in which the type (ii) neighborhoods $Y$ of $x$ we obtain when $\square_{\mathrm{O}} \alpha \in x$ (for $|\alpha| \neq \varnothing$ ) by setting $Y=|\alpha| \backslash\{f(|\alpha|)\}$ are thereby guaranteed not to be formula-definable. We need this because otherwise $Y$ could be $|\beta|$ for some formula $\beta$ which would require that we had $\mathrm{O} \beta \in x$ : but when $\alpha$ is consistent there is no need for any such 
$\mathrm{O} \beta$ (where $\vdash \beta \rightarrow \alpha$, since $|\beta|$ would then be included in $|\alpha|$ ) to belong to $x$. To reduce congestion, we write $f(\gamma)$ from now on, instead of $f(|\gamma|)$.

Lemma 3.3 For all formulas $\beta, \gamma$, if $|\gamma| \backslash\{f(\gamma)\} \subseteq|\beta|$, then $|\gamma| \subseteq|\beta|$.

Proof: Since the conclusion is automatic for $|\gamma|=\varnothing$, we may assume that $|\gamma| \neq \varnothing$. In this case $f(\gamma) \in|\gamma|$, and $|\gamma| \backslash\{f(\gamma)\}$ is a proper subset of $|\gamma|$; if this set is included in $|\beta|$ while $|\gamma|$ is not, we must have $|\gamma \wedge \neg \beta|=\{f(\gamma)\}$, contradicting Lemma 3.1 .

We are now in a position to prove the Fundamental Theorem identifying truth and membership for the present version of canonical models. The part of the proof dealing with O-formulas is standard (cf. [1], [14]).

Theorem 3.4 For all formulas $\alpha$ and all $x \in W$, where $\langle W, N, V\rangle$ is the canonical model, $\alpha \in x$ if and only if $\langle W, N, V\rangle \models_{x} \alpha$.

Proof: By induction on the complexity of $\alpha$, we show that the result holds for all $x \in W$. We deal only with the inductive cases in which $\alpha$ is of the form $\mathrm{O} \beta$, and in which $\alpha$ is of the form $\square_{\mathrm{O}} \beta$. Since $\beta$ is of lower complexity than $\alpha$, the inductive hypothesis allows us to assume that $|\beta|=\|\beta\|$.

First, suppose $\alpha=\mathrm{O} \beta$. We must show that $\mathrm{O} \beta \in x \Longleftrightarrow \models_{x} \mathrm{O} \beta$ (suppressing the reference to the model here), i.e., using the inductive hypothesis, that $\mathrm{O} \beta \in x \Longleftrightarrow$ | $\beta \mid \in N_{x}$. The $\Rightarrow$ direction follows from part (i) of the definition of $N$. For the $\Leftarrow$ direction, suppose that $|\beta| \in N_{x}$. Since only type (i) neighborhoods of $x$ are formuladefinable (Lemma 3.2), this means that $|\beta|=|\gamma|$ for some $\gamma$ with $\mathrm{O} \gamma \in x$. Since $|\beta|=|\gamma|, \vdash \beta \longleftrightarrow \gamma$, whence by (Cong. O), $\vdash \mathrm{O} \beta \longleftrightarrow \mathrm{O} \gamma$, which implies that $\mathrm{O} \beta \in x$.

Next, suppose that $\alpha=\square_{\mathrm{O}} \beta$. Again, what we have to show is that $\square_{\mathrm{O}} \beta \in$ $x \Longleftrightarrow Y \subseteq|\beta|$, for some $Y \in N_{x}$. First, for the $\Rightarrow$ direction, suppose $\square_{\mathrm{O}} \beta \in x$.

Case 1: $|\beta| \neq \varnothing$. Then $f(\beta) \in|\beta|$ and so since $\square_{\mathrm{O}} \beta \in x,|\beta| \backslash\{f(\beta)\} \in N_{x}$, by part (ii) of the definition of $N$. Since this is a subset of $|\beta|$, it serves as the desired $Y$.

Case 2: $|\beta|=\varnothing$. Then $\vdash \beta \rightarrow \perp$, and thus $\vdash \square_{\mathrm{O}} \beta \rightarrow \square_{\mathrm{O}} \perp$, by (Mon. $\square_{\mathrm{O}}$ ). Therefore, since $\square_{\mathrm{O}} \beta \in x$, we have $\square_{\mathrm{O}} \perp \in x$, in which case, by (A2) $\mathrm{O} \perp \in x$. So by part (i) of the definition of $N, \varnothing \in N_{x}$ and we may take $\varnothing$ as the desired $Y$.

Turning to the $\Leftarrow$ direction, suppose that for some $Y \in N_{x}, Y \subseteq|\beta|$. We must show $\square_{\mathrm{O}} \beta \in x$.

Case 3: $\quad Y$ is a type (i) neighborhood of $x$. Thus, $Y=|\gamma|$ for some $\gamma$ with $\mathrm{O} \gamma \in x$. Since $|\gamma| \subseteq|\beta|, \vdash \gamma \rightarrow \beta$, so by (Mon. $\square_{\mathrm{O}}$ ), $\vdash \square_{\mathrm{O}} \gamma \rightarrow \square_{\mathrm{O}} \beta$. But $\square_{\mathrm{O}} \gamma \in x$, since $\mathrm{O} \gamma \in x$ (by A1); therefore $\square_{\mathrm{O}} \beta \in x$.

Case 4: $\quad Y$ is a type (ii) neighborhood of $x$, in which case $Y=|\gamma| \backslash\{f(\gamma)\}$ for some formula $\gamma$ for which $|\gamma| \neq \varnothing$ and for which $\square_{\mathrm{O}} \gamma \in x$. Thus the supposition that $Y \subseteq|\beta|$ is the supposition that $|\gamma| \backslash\{f(\gamma)\} \subseteq|\beta|$, and we appeal to Lemma 3.3 to conclude that $|\gamma| \subseteq|\beta|$; so $\vdash \gamma \rightarrow \beta$, whence by (Mon. $\square_{\mathrm{O}}$ ), $\vdash \square_{\mathrm{O}} \gamma \rightarrow \square_{\mathrm{O}} \beta$, so since $\square_{\mathrm{O}} \gamma \in x$, we have $\square_{\mathrm{O}} \beta \in x$ as desired. 
In the manner familiar from the references already cited (coupled with the earlier observations of soundness), Theorem 3.4 gives us:

Corollary 3.5 For any formula $\alpha, \vdash \alpha$ if and only if $\alpha$ is valid on every frame $\langle W, N\rangle$.

4 Two extensions of the basic system Given our original starting point, with $\mathrm{O}$ as the epistemic $\mathrm{K}$, it is of some interest to consider such additional axioms as (A3) and (A4), versions of the familiar T-schema for each of our primitive operators.

$$
\begin{aligned}
& \mathrm{O} \alpha \rightarrow \alpha . \\
& \square_{\mathrm{O}} \alpha \rightarrow \alpha .
\end{aligned}
$$

The two systems we consider here are the results of appending in the first place (A3) to the axiomatization of the basic system whose completeness was proved abovecall this the first extended system — and, secondly, just (A4) to that axiomatization, 'the second extended system'. In view of (A1) $\left[=\mathrm{O} \alpha \rightarrow \square_{\mathrm{O}} \alpha\right]$, (A3) can be derived, given the basic system, from (A4); so the first extended system is a subsystem of the second (a proper subsystem, as will emerge in the following section). We begin by considering this second system. We shall show that it is sound and complete with respect to the class of frames $\langle W, N\rangle$ satisfying, predictably enough (cf. [1], pp. 224 and 262), the condition

(t) For all $x \in W$, if $Y \in N_{x}$ then $x \in Y$.

For soundness, it suffices to check that every instance of the schema (A4) is valid on any frame satisfying $(\mathrm{t})$.

The completeness proof cannot proceed exactly as in the preceding section, however, because we shall need to make sure that when we remove an element from nonempty $|\alpha|$ for which $\square_{\mathrm{O}} \alpha \in x$ to obtain a type (ii) neighborhood of $x$, the point removed is not $x$ itself: this would stop us from verifying that $(\mathrm{t})$ is satisfied by the frame of the resulting model. Accordingly, we need a variant of the function $f$ employed above: our choice function (we shall write $g(x, \alpha)$ again, rather than $g(x,|\alpha|)$ ) will need to be sensitive not only to $\alpha$ but also to $x$. This new function, $g$, is defined for all pairs $x, \alpha$, and we stipulate (as our "selection" requirement) that whenever $|\alpha| \neq \varnothing, g(x, \alpha)$ is some element, other than $x$, of $|\alpha|$. If $x$ does not belong to $|\alpha|$ in the first place, there is no problem finding $g(x, \alpha)$ satisfying this requirement that $g(x, \alpha) \neq x$, since $|\alpha| \neq \varnothing$. On the other hand if $x \in|\alpha|$, we know by Lemma 3.2 that $|\alpha| \backslash\{x\}$ is nonempty (since otherwise it would be formula-definable, as $|\perp|)$, so $g(x, \alpha)$ can select some element of $|\alpha| \backslash\{x\}$, and again our requirement that $g(x, \alpha) \neq x$ is satisfied. We repeat Lemma 3.3 for the new selection function, as Lemma 4.1. its proof is exactly as for Lemma 3.3. substituting $g(x, \gamma)$ for $f(\gamma)$.

Lemma 4.1 For all $x \in W$ and all formulas $\beta$, $\gamma$, if $|\gamma| \backslash\{g(x, \gamma)\} \subseteq|\beta|$, then $|\gamma| \subseteq|\beta|$.

We now define the canonical model for what we are calling the second extended system (the basic system + A4). We continue to write $\langle W, N, V\rangle$, with $W$ and $V$ defined as before, though now understanding maximal consistency relative to the new system. $N_{x}$, for $x \in W$, again comprises sets of two types: type (i) neighborhoods, of the form 
$|\alpha|$ for $\alpha$ such that $\mathrm{O} \alpha \in x$, and type (ii) neighborhoods, of the form $|\alpha| \backslash\{g(x, \alpha)\}$ for $\alpha$ such that $\square_{\mathrm{O}} \alpha \in x$ and $|\alpha| \neq \varnothing$. Theorem 3.4 continues to hold, reinterpreted for the present canonical model; a proof mimics the original proof, using Lemma 4.1 in place of Lemma 3.3. As a corollary, we have (soundness and) completeness with respect to the class of frames satisfying (t); the proof is relatively straightforward (using the new $g$ function for the if direction).

Corollary 4.2 For any formula $\alpha, \alpha$ is provable in the second extended system if and only if $\alpha$ is valid on every frame $\langle W, N\rangle$ satisfying $(t)$.

5 Incompleteness of the first extension The second of the two extensions of the basic system introduced in the preceding section is determined, as we saw there (Corollary 4.2), by the class of all frames $\langle W, N\rangle$ satisfying the condition ( $\mathrm{t}$ ), where determined by has the same meaning as sound and complete with respect to. In this section, we shall show, by contrast, that the first extension is incomplete on the present semantics, in the sense of being determined by no class of frames (Theorem 5.4 below). This is established by showing that although every frame validating (A3) validates (A4), (A4) is not derivable with the aid of truth-functional logic alone, (A1), (A2), (Cong. O), and (Mon. $\square_{\mathrm{O}}$ ) from (A3)—which is to say that (A4) is not provable in the first extended system. In fact, (A3) and (A4), though not interdeducible in the field of the basic system, are valid on precisely the same frames, namely those satisfying ( $\mathrm{t}$ ); we need only part of this (Lemma 5.2 below) for present purposes. The proof of Lemma 5.1 s straightforward; for Lemma 5.2 use Lemma 5.1.

Lemma 5.1 Every frame validating (A3) satisfies (t).

Lemma 5.2 Every frame validating (A3) validates (A4).

To show that (A4) is not provable in the first extension of the basic system, we introduce another extension - we shall call it $S$ - of the basic system and show very simply that (A4) is not provable in $S . S$ is to be the smallest normal bimodal logic in each of $\mathrm{O}$ and $\square_{\mathrm{O}}$, containing all instances of (A1), (A2), and (A3). Working with birelational Kripke frames $\left\langle W, R_{O}, R_{\square}\right\rangle$ in which $R_{O}$ and $R_{\square}$ are binary relations on $W$ interpreting $\mathrm{O}$ and $\square_{\mathrm{O}}$ respectively in the usual manner (i.e., by universal quantification over appropriately related points), one obtains by standard methods the result that $S$ is determined by the class of all such frames in which the following three conditions are satisfied.

(a1) $R_{\square} \subseteq R_{O}$.

(a2) $R_{\square}(x)=\varnothing \Rightarrow R_{O}(x)=\varnothing$ (for all $x \in W$ ).

(a3) $R_{O}$ is reflexive.

In (a2) the ' $R(x)$ ' notation denotes the set of all $y \in W$ such that $R x y$, for $R=R_{\square}$, $R_{O}$; the conditions (a1), (a2), and (a3) correspond respectively to (A1), (A2), and (A3).

\section{Lemma $5.3 \quad(A 4)$ is not provable in $S$.}

Proof: Consider the frame $\left\langle W, R_{O}, R_{\square}\right\rangle$ in which $W$ is the set of integers and $R_{\mathrm{O}}$ and $R_{\square}$ are respectively the relations $\leq$ and $<$. The conditions (a1), (a2), and (a3) 
are satisfied —in the case of (a2), because the antecedent $R_{\square}(x)=\varnothing$ never holds—so every theorem of $S$ is valid on this frame. Clearly, however, since $R_{\square}$ is not reflexive, not every instance of (A4) is valid on the frame (e.g., take $\alpha$ in $\square_{\mathrm{O}} \alpha \rightarrow \alpha$ as $p_{1}$.)

As a corollary, we have

Theorem 5.4 There is no class of frames $\langle W, N\rangle$ with respect to which the first extended system is sound and complete.

6 Some reflections on the incompleteness example The example of incompleteness presented by Theorem 5.4 is of some theoretical interest because of a contrast with a general result of Lewis 9 formulated here with the notion of a noniterative schema, by which is meant a schema in which no modal operator occurs within the scope of a modal operator: the smallest congruential multimodal logic containing all instances of some set of noniterative schemata is determined by the class of all neighborhood frames validating all those schemata. The neighborhood frames spoken of here have one neighborhood-assigning function for each of the primitive modal operators, truth in a model on such a frame being defined with the aid of a clause for each operator, treating it via the neighborhood-assigning function as $\mathrm{O}$ is treated via $N$ in the semantics of Section $2 .{ }^{6}$ Now the point of interest here is that (A1), (A2), and (A3) are noniterative schemata, so at first sight Lewis's result would seem to contradict Theorem 5.4 nnce we observe that (Mon. $\square_{\mathrm{O}}$ ) can be replaced, without alteration to the logic axiomatized, by a congruentiality rule for $\square_{\mathrm{O}}$ together with the schema $\square_{\mathrm{O}}(\alpha \wedge \beta) \rightarrow \square_{\mathrm{O}} \alpha$-another noniterative schema. But the contradiction is only apparent, since our semantics has not been a multiple neighborhood semantics of the kind addressed by that result.

To convert the semantic apparatus of Section 2 above to that which Lewis had in mind, we must use frames with two neighborhood-assigning functions, one for $\mathrm{O}$ (which we continue to denote by $N$ ) and one for $\square_{\mathrm{O}}$ (which we shall denote by $M$ ), defining truth at a point in a model $\langle W, N, M, V\rangle$ on such a frame with the aid of the clause given in that section for $O$, but replacing the treatment there offered for $\square_{\mathrm{O}}$ with the following (analogous) clause.

$$
\langle W, N, M, V\rangle \models_{x} \square_{\mathrm{O}} \alpha \text { if and only if }\|\alpha\| \in M_{x} .
$$

Frames $\langle W, N, M\rangle$ for the basic system must now satisfy the following conditions.

(i) $X \subseteq Y$ and $X \in M_{x}$ imply $Y \in M_{x}$, for all $x \in W$, all $X, Y \subseteq W$.

(ii) $\quad N_{x} \subseteq M_{x}$, for all $x \in W$.

(iii) $\varnothing \in M_{x}$ implies $\varnothing \in N_{x}$.

A routine argument shows that the basic system is determined by the class of all frames $\langle W, N, M\rangle$ satisfying (i), (ii), and (iii). Our present interest, however, is in the first extension of the basic system, so we need to observe that (A3) is valid on $\langle W, N, M\rangle$ just in case this frame satisfies the condition (t) from Section 4, and that a similar relation holds with regard to (A4) and the condition—call it $\left(\mathrm{t}^{\prime}\right)$ - that results from (t) by replacing $N$ by $M$ :

$\left(\mathrm{t}^{\prime}\right) \quad$ For all $x \in W$, if $Y \in M_{x}$ then $x \in Y$. 
It is not hard to see that $\left(\mathrm{t}^{\prime}\right)$ would follow from $(\mathrm{t})$ with the aid of the $\Rightarrow$ direction of the following condition, which in effect reinstates in the present multiple neighborhood semantics the clause for $\square_{\mathrm{O}}$ from Section 2:

$$
\text { (*) } Y \in M_{x} \Longleftrightarrow \exists X \in N_{x} . X \subseteq Y \text {. }
$$

The trouble is, however, that it is only the $\Leftarrow$ direction of $(*)$ that is secured by the conditions our current frames must satisfy for them to validate the axioms of the first extended system. (In fact, this direction of $(*)$ follows from two of those conditions, namely (i) and (ii).) To check that the remaining conditions do not help in obtaining $(*)$, it suffices to show, in view of what has just been seen with $(\mathrm{t})$ and $\left(\mathrm{t}^{\prime}\right)$, that there are frames $\langle W, N, M\rangle$ satisfying (i), (ii), (iii), and (t) which do not satisfy $\left(\mathrm{t}^{\prime}\right)$. But we can simply rework the example of the birelational Kripke frame $\left\langle W, R_{\mathrm{O}}, R_{\square}\right\rangle$ from the proof of Lemma 5.3 to this end, putting $N_{x}=\left\{R_{\mathrm{O}}(x)\right\}$ and $M_{x}=\left\{R_{\square}(x)\right\}$ for all $x \in W$.

7 Closing comments The preceding section serves as a reminder that whether a modal logic is incomplete in the apparently absolute sense of not being determined by any class of frames is itself relative (perhaps better: sensitive) to the semantic apparatus in play - to the conception of frame and the definition of truth at a point in a model on such a frame, that is. Relative to the semantic apparatus of Section 2, our first extension of the basic system is incomplete, while relative to the multiple neighborhood semantics, that extension is complete.

Returning to the basic system and to the motivation for considering it (and its extensions) offered in Section 1, one natural next step would be to consider the question of an analogous basic system for a congruential-to-normal bimodal logic. The justification in Section 2 for the semantic treatment of $\square_{O}$ there proposed (that for $O$ remaining intact) adapts to a justification for the clause

$$
\langle W, N, V\rangle \models{ }_{x} \square_{\mathrm{O}} \alpha \text { if and only if } \cap N_{x} \subseteq\|\alpha\| .
$$

The obvious question then arises: How do we axiomatize the class of formulas valid on every frame when this clause is employed in the truth-definition? (Conspicuously, instances of (A2) are, in general, no longer among such formulas.) Somewhat further afield, one might consider a trimodal logic which was, as we might say, (minimally) congruential-to-monotone-to-normal. Here we should need two additional primitive operators alongside $\mathrm{O}$, one for the monotone extension, $\square_{\mathrm{O} m}$ say, and one for the normal extension, $\square_{\mathrm{O} n}$. The rough and ready glosses would be, with the epistemic reading of O: $\square_{\mathrm{O} m} \alpha$ means that it follows from some proposition known by the subject that $\alpha$, while $\square_{\mathrm{On}}$ means that it follows from some set of propositions known by the subject that $\alpha$. Clearly, the latter operator can be regarded as representing the normal extension not only of $\mathrm{O}$, but, equally well, of $\square_{\mathrm{Om}}$. We shall not, however, investigate these matters further here.

Acknowledgments I am grateful to graduate students Thomas Bull and Brian Weatherson for technical corrections to an earlier draft of this material, as well as to my colleagues Alan Crooke and Richard Holton (see Note 3). 


\section{NOTES}

1. On p. 38 of [6], Hintikka mentions the possibility (though in the end he is not very enthusiastic about it) of reading his ' $\mathrm{K}_{a} p$ ' as 'It follows from what $a$ knows that $p$ '. Similarly, with a view to sidestepping monotony objections in boulomaic logic on p. 409 of 7 we find, as a suggested alternative to reading ' $W_{a} p$ ' as ' $a$ wants that $p$ ': $a$ has desires whose satisfaction entails that $p$. (Epistemic applications are also considered in [7].) Similarly, with a rather syntactical conception of propositions in mind, Williamson (17], p. 85) suggests that the epistemic operator $\mathrm{K}$ be supplemented with another, which he writes as $\mathrm{K}^{-}$, the intended reading of $\mathrm{K}^{-} p$ being 'For some $q$ of which $p$ is a conjunct, $\mathrm{K} q$ '.

2. Lewis 11] gives a somewhat different treatment-foreshadowed in Lewis 10 according to which the possibility here legitimately ignored (when the proposition that it does not obtain is, as Hanfling says, "taken for granted") in normal contexts, may no longer be properly ignored with the change of context brought about by explicitly raising that very possibility. This allows him to retain monotony (indeed normality) for any given context. References to further non-monotone treatments (Dretske, Nozick, etc.) may be found in Note 8 of [4]; often the formulations of epistemologists (in terms of closure) do not distinguish the conditions of monotony and regularity. A noteworthy exploration of (specifically) epistemic logic without the problematic conditions may be found in [13.

3. The multiplicity of distinct notions going by the name of epistemic possibility current in philosophical discussion was drawn to my attention by my colleague Alan Crooke, conversation with whom influenced several aspects of this paper, beginning with its opening sentence. Crooke has noted that doxastic possibility is sometimes confusedly referred to as epistemic possibility; on p. 55 of [12], the epistemically possible seems to be identified not with what is not known to be false, but with what is not known a priori to be false. I thank Richard Holton for this reference. Putnam purports to be summarizing Kripke, and puts scare-quotes around the phrase 'epistemically possible'. Still keeping to one side the issues of regularity and monotony, there is the matter of whether more is required than is given by "not known not to be the case" formulations: this was argued by Hacking [3], who thinks of himself as picking up where the originator of talk of epistemic possibility-G. E. Moore-left off, but is contested in Teller [16] and Williamson[17].

4. The fourth option is mentioned in Note 2 of Kaplan [8], where E-whispering is introduced as the congruential extension of whispering; in this formulation, one operator is an extension of another (relative to a given logic) if the result of prefixing the latter to any formula follows (according to the logic) from the result of prefixing the former to that formula. Kaplan's own formulation reads: "To notions for which substitution under logical equivalence is not correct, such as 'she whispered that,' we may associate an E-analog, 'she whispered that,' obtained by closing the notion in question under logical equivalence" ([8], p. 49).

5. This notion of definability by a formula is of course to be distinguished from the definability of a class of frames by a formula which obtains when the frames validating the formula are precisely those in the class.

6. In fact Lewis works with a different formulation of the semantics which is, however, equivalent to the many-neighborhoods version envisaged here. See [1], p. 211, Ex.7.10, or - where the equivalence was perhaps first noted $-\$ 3$ of [5]. 


\section{REFERENCES}

[1] Chellas, B. F., Modal Logic: An Introduction, Cambridge University Press, Cambridge,

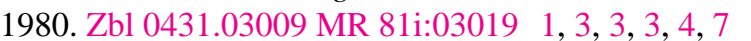

[2] Chellas, B. F., and A. McKinney, "The completeness of monotonic modal logics," Zeitschrift für mathematische Logik und Grundlagen der Mathematik, vol. 21 (1975), pp. 379-383. Zbl 0331.02007 MR 51:12480 1

[3] Hacking, I., "Possibility," Philosophical Review, vol. 76 (1967), pp. 143-168.

[4] Hanfling, O., "How is scepticism possible?," Philosophy, vol. 62 (1987), pp. 435-453. 1,7

[5] Hansson, B., and P. Gärdenfors, "A guide to intensional semantics," pp. 151-167 in Modality, Morality and Other Problems of Sense and Nonsense: Essays Dedicated to Sören Halldén, CWK Gleerup Bokforlag, Lund, 1973. MR 56:11757 7

[6] Hintikka, J., Knowledge and Belief, Cornell University Press, Ithaca,1962.

[7] Humberstone, I. L., "The formalities of collective omniscience," Philosophical Studies, vol. 48 (1985), pp. 401-423. MR 87d:03043 7,7

[8] Kaplan, D., "A problem in possible-world semantics," p. 41-52 in Modality, Morality, and Belief: Essays in Honor of Ruth Barcan Marcus, edited by W. Sinnott-Armstrong et al., Cambridge University Press, Cambridge, 1995. 7,7

[9] Lewis, D., "Intensional logics without iterative axioms," Journal of Philosophical Logic, vol. 3 (1974), pp. 457-466.Zbl 0296.02014|MR 54:9981 6

[10] Lewis, D., "Scorekeeping in a language game," Journal of Philosophical Logic, vol. 8 (1979), pp. 339-359.

[11] Lewis, D., "Elusive knowledge," Australasian Journal of Philosophy, vol. 74 (1996), pp. 549-567.

[12] Putnam, H., "Is water necessarily $\mathrm{H}_{2} \mathrm{O}$ ?," Chapter 4 in Putnam, Realism with a Human Face, edited by J. Conant, Harvard University Press, Cambridge, 1990.

[13] Schotch, P. K., and R. E. Jennings, "Epistemic logic, skepticism, and non-normal modal logic," Philosophical Studies, vol. 40 (1981), pp. 47-67.MR 82j:03022 7

[14] Segerberg, K., An Essay in Classical Modal Logic, vol. 1, Uppsala Universitet, Uppsala, 1971. Zbl 0311.02028 MR 49:4756 1.3.3.3.3

[15] Segerberg, K., Classical Propositional Operators, Clarendon Press, Oxford, 1982. Zbl 0491.03003||MR 83i:03001 1

[16] Teller, P., "Epistemic possibility," Philosophia, vol. 2 (1972), pp. 303-320.

[17] Williamson, T., "Verificationism and non-distributive knowledge," Australasian Journal of Philosophy, vol. 71 (1993), pp. 78-86. 7.7

Department of Philosophy

Monash University

Wellington Road

Clayton, Victoria 3168

AUSTRALIA

email: Lloyd.Humberstone@arts.monash.edu.au 